\title{
Decarbonising the Finnish Transport Sector by 2050: Electricity or Biofuels?
}

\author{
Skytte, Klaus; Bramstoft Pedersen, Rasmus
}

Published in:

The Nexus: Energy, Environment and Climate Change

Link to article, DOI:

10.1007/978-3-319-63612-2_1

Publication date:

2018

Document Version

Peer reviewed version

Link back to DTU Orbit

Citation (APA):

Skytte, K., \& Bramstoft Pedersen, R. (2018). Decarbonising the Finnish Transport Sector by 2050: Electricity or Biofuels? In The Nexus: Energy, Environment and Climate Change (pp. 3-22). Springer. Green Energy and Technology https://doi.org/10.1007/978-3-319-63612-2_1

\section{General rights}

Copyright and moral rights for the publications made accessible in the public portal are retained by the authors and/or other copyright owners and it is a condition of accessing publications that users recognise and abide by the legal requirements associated with these rights.

- Users may download and print one copy of any publication from the public portal for the purpose of private study or research.

- You may not further distribute the material or use it for any profit-making activity or commercial gain

- You may freely distribute the URL identifying the publication in the public portal 


\title{
Decarbonising the Finnish transport sector by 2050 - electricity or biofuels?
}

\author{
Klaus Skytte and Rasmus Bramstoft Pedersen \\ Technical University of Denmark, Kgs. Lyngby 2800, Denmark \\ Email: klsk@dtu.dk and rabpe@dtu.dk
}

Keywords energy system modelling, transport, electric vehicles, biofuels, STREAM model

\begin{abstract}
Finland has set ambitious long-term targets which aim to reduce greenhouse gas emissions from the transport sector and the whole energy system by 2050. By utilising the energy system model STREAM, which includes the power, heat and transport sectors, this paper develops two alternative scenarios for the transport sector by 2050 - one with a high percentage of electric vehicles (EV) and another with a high percentage of biofuels (BIO), and compares the scenario results with a known Carbon-Neutral Scenario (CNS) which is adopted from the Nordic Energy Technology Perspective (IEA 2013a). The socio-economic value of the total system cost is computed and the system integration of the transport sector with the electricity and heating sectors is simulated with an hourly time resolution.
\end{abstract}

This study finds that a Finnish transport sector with a high share of EV by 2050 leads to the lowest total annual system cost of the scenarios and yields a reduction by $2.3 \%$ compared to CNS. While the transport configuration in the BIO scenario achieves the highest total annual system cost which is $0.4 \%$ higher than CNS. The robustness of the results is tested through a sensitivity analysis which shows that the costs (investment and maintenance) of biodiesel cars and $\mathrm{EV}$ are the most sensitive parameters in the comparative analysis of the scenarios. 


\section{Introduction}

In recent years, reduction of greenhouse gas (GHG) emissions from the power, heat and transport sectors have received worldwide attention. To mitigate climate changes, both national and international agreements on climate and energy related targets have been stated. Finland has set ambitious targets which aims to reduce the GHG emissions by $20 \%$ by 2020 and 80-95\% by 2050 compared to the 1990 level (EC 2011, IEA 2013b). As an intermediate target, Finland has the ambition of integrating renewable energy sources (RES) in the energy system that accounts for 38\% of the gross final energy consumption by 2020 (IEA 2013a).

In EU, a target share of at least 10\% RES in the energy used in the transport sector by 2020 is stated (EC 2009). In 2011 the Finnish transport sector accounted for approximately 20\% of the national GHG emissions (FMEE 2014, IEA 2013a). As stated in the Finnish national and climate strategy, emissions from the transport sector can be reduced by $15 \%$ by 2020 and up to $35-40 \%$ by 2030 compared to the 2005 level (FMEE 2014). Finland has, furthermore, a notably goal of implementing 20\% renewables in the road transport by 2020 (IEA 2013a).

The Finnish power and heating system will experience a remarkable transition in the future, heading towards power and heat production based on carbon neutral energy sources.

Currently the Finnish electricity generation portfolio is very diverse, and is primarily supplied by fossil fuels and nuclear. However, the future power generation portfolio in Finland is expected to rely on nuclear and forestry-related renewables (IEA 2013b). The share of electricity generated by nuclear power plants is expected to increase to the double by 2025 , since the Finnish Parliament has approved new nuclear power plants to be constructed (IEA 2013b). Finland is the most forested country in Europe, hence there is a major potential of incorporating a large share of forestry-related renewables in the energy system. Biomass can be used in the power, heating and transport sector and will therefore play a prominent role in the future energy system (IEA 2013b).

Currently, the transport sector is highly dependent on oil-based fossil fuels i.e. diesel and gasoline. Hence, radical restructuring of the fuel use and vehicle stock is required in order to reduce the GHG emissions in transport sector. This restructuring has to be carried out by 
introducing governmental initiatives in combination with improving technological development in the non-fossil technologies and enhancing infrastructure.

The transition towards a green transport sector by 2050 is related to stronger synergies with the power and heating sector prompted by higher utilisation of electric and biofuel transportation. Hence, for analysing the transport sector, a complete analysis of the entire energy system, including the power, heat and transport system is required.

By using the simulation model STREAM (Münster et al. 2014), a holistic analysis of the transport scenarios and their system integration with the electricity and heating sectors will be conducted. Two scenarios for a fossil free Finnish transport sector by 2050 are developed. The first scenario (EVS) includes a high percentage of electric vehicles (EV) and the second scenario (BIOS) includes a high percentage of biofuel-use in the transport sector.

\section{Previous studies:}

Extensive studies considering a complete energy system analysis including the transport sector has been conducted. Nordic Energy Technology Perspective 2013 provides pathways to a Carbon Neutral Energy Future by 2050 for the Nordic countries (IEA 2013a). The entire energy system in the Nordic countries is described by a Carbon Neutral Scenario (CNS). In this paper, CNS represents the reference scenario and it is used to carry out a comparative evaluation of the two alternative transport scenarios.

Research on benefits for the power system and the customers has been carried out within the field of integrating a larger share of EV in the energy and transport system. The concept of vehicles-to-grid (V2G) has been explained, and the potential benefits of implementing V2G in the energy system have been investigated (Kempton \& Tomic 2005). The influence of the power system investments when integrating a larger share of the variable renewable energy (VRE) source wind in combination with plugin electric vehicles, and heat storages has been studied (Kiviluoma \& Meibom 2010). The results pointed to the findings that EV can store the electricity for later use and thus can increase the flexibility of the power system.

Furthermore, the optimal configuration of an integrated power and transport system has been identified by utilising a linear optimisation model (Juul \& Meibom 2011). 
Comprehensive studies have been conducted considering a larger implementation of bioenergy and biofuels in the energy and transport system (e.g. Skytte et al. 2006). Börjesson et al. (2013) presented a review of studies which investigate biofuels future in the transport sector by utilising energy economic modelling. The study is comprehensive and finds that the future market share of biofuels in the transport sector varies significantly in the review studies. They find that biofuels plays a key role in the medium-term, while most of the reviewed studies favour hydrogen or electricity in the longer-term.

\section{The STREAM model}

The different transport scenarios are implemented in the energy system model STREAM (Sustainable Technology Research and Energy Analysis Model), which is a simulation tool that includes the complete energy system. The model provides an overview of the energy flow across all sectors, from primary fuel through conversion technologies to the end-users. Furthermore, STREAM allows simulating different scenarios in order to perform comparative analyses of their solutions, considering the electricity, heat and transport sectors simultaneously (Connolly et al. 2010, Skytte and Skjerk Christensen 1999).

The modelling tool consists of two sub-models i.e. the energy flow model and the duration curve model, as illustrated in Figure 1, both of which relies on a bottom-up approach. Even though STREAM is not an optimisation model, the operation of the energy system is optimised under the given conditions.

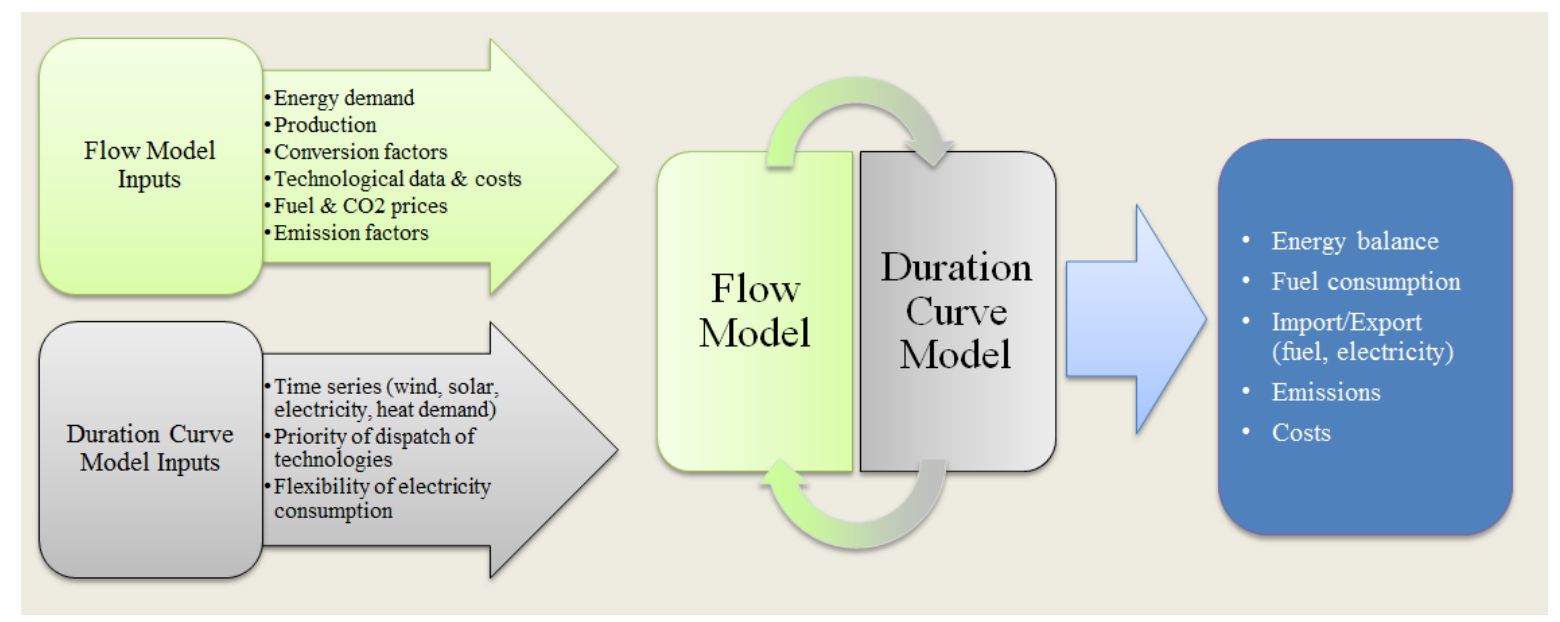

Figure 1: Linkage between Flow Model and Duration Curve Model in STREAM 
The model working flow and interactions of the two sub-models in STREAM is as follows (Münster et al. 2014):

- The Energy Flow Model provides an overview of demand and supply in a given year. Projection of demands for energy services is specified and consequently the final energy consumption is calculated, according to the defined inputs for supply. Finally, an overview of GHG emissions, energy resources and fuel conversion is created.

- The Duration Curve Model computes the system balancing on an hourly time resolution. It includes correlations in the electricity and district heating systems: uncontrollable vs. dispatchable electricity, combined heat and power, district heating generation, storage facilities, flexible and non-flexible demand, etc. The results computed in the Duration Curve Model is latter used as input is to the Energy Flow Model in an iterative procedure.

In STREAM, the transport system is modelled as follows. The transport system is divided into four independent sectors: passenger, freight, agriculture and fishery. The current transport work (passenger-kilometre, tonne-kilometre and energy consumption in agriculture and fishery) is specified. The projection for the demand in the year of simulation is computed by defining metrics for expected economic growth and specific energy intensity factors. Driven by the computed demand, the transportation sectors are described along with their synergies with the energy system. Furthermore, the transport work is allocated by vehicle type (e.g. car, bus, train, plane, bike, etc.), the utilisation degree (or stocking density), the composition of fuels for transportation means for both passenger and freight transport along with agriculture and fishery is defined according to the purpose of the scenario. Associated costs and emissions related to fuel production and consumption as well as vehicle acquisition and maintenance are accounted for. Finally, modelling of flexible fuel production and charging of EV are facilitated by the Duration Curve model.

\section{Main data assumptions}

To perform the quantitative assessment, metrics for the electricity and heat sector along with specific prices are adopted from the anticipative Carbon-Neutral Scenario (CNS) outlined in the NETP 2013 project (IEA 2013a). CNS push beyond the IEA's global two Degree Scenario (2DS) (IEA 2012), towards a near carbon-neutral energy system by 2050. CNS 
takes into account interactions between countries while hold promise of achieving the national energy policy targets. Furthermore, CNS representing a pathway which can potentially reduce the aggregated emissions by $85 \%$ by 2050 compared to 1990 levels.

Data for generation technologies and costs for vehicles (DEA 2013) are adopted from publicly available catalogues (DEA 2012, 2013).

Fossil and biofuel prices along with the $\mathrm{CO}_{2}$ price are parameters that potentially have a sustainable influence on the final results. The price levels by 2050 of these parameters are presented in Table 1.

The price projections for fossil fuels i.e. hard coal, crude oil, and natural gas is adopted from NETP 2013 (IEA 2013a). The projections for biofuel prices are adopted from EA Energy Analyses (EA 2013) and are an output from the Global Assessment Model (GCAM). The $\mathrm{CO}_{2}$ price reflects the marginal abatement costs in the electricity system and show a steep increment in price towards 2050 aided by the ambitious and strict energy policy targets in the 2DS.

Table 1: $\quad$ Fuel and $\mathrm{CO}_{2}$ prices by 2050 which are implemented in STREAM.

\section{Fuel prices}

\begin{tabular}{lll}
\hline Natural Gas & 6.02 & $€ / \mathrm{GJ}$ \\
Nuclear - Uranium & 4.00 & $€ / \mathrm{GJ}$ \\
Biomass (Straw, woodwaste) & 9.10 & $€ / \mathrm{GJ}$ \\
\hline Biomass (Energy crops ) & 9.78 & $€ / \mathrm{GJ}$ \\
Biomass (manure) & 0.00 & $€ / \mathrm{GJ}$ \\
\hline Imported electricity & 31.13 & $€ / \mathrm{GJ}$ \\
Coal & 1.58 & $€ / \mathrm{GJ}$ \\
\hline Oil & 16.40 & $€ / \mathrm{GJ}$ \\
\hline $\mathrm{CO}_{2}$ price & 120.30 & $€ / \mathrm{CO} 2$ \\
\hline
\end{tabular}




\section{Description of the 2050 Scenarios}

In this study two alternative transport scenarios towards a fossil free transport sector will be investigated, namely, EVS and BIOS, and compared with CNS. In NETP 2013, CNS contains a very diverse fuel mix in the transport sector, also including different biofuels. However, in this paper, the preferred biofuels are biodiesel and UPGR biogas.

The transport sector is currently almost independent of the power and heat generation, however, in the future their synergies will be stronger. Thus restructuring of the fuel use in the transport sector has an influence at the entire energy system. Heading towards a transport sector with a higher share of EV implies increased electricity consumption. Furthermore if EV relies on the concept of G2V, demand side flexibility is provided to the system.

In this paper the power, district heat production and transport sector is implemented based on the following assumptions:

- The electricity system is designed in order to ensure consistencies in the final energy production between the two alternative transport scenarios and CNS, except for the electricity production by wind turbines. In EVS onshore wind is increased in order to cover the increased electricity demand, while in BIOS offshore wind production is reduced in order to meet the demand.

- In the district heating and industry sectors, the percentages allocation of the heating supply mix as in CNS are adopted in the STRAM model, thus inconsistencies in the actual production may appear.

- The transport sector is designed according to the purpose of the alternative scenarios i.e. EVS or BIOS.

\section{Resources}

The chain from primary resources to the end consumers and thus the relation between the availability of resources and the service provided to the consumers is essential to investigate in order to evaluate the degree of self-sufficiency. By applying STREAM which covers the complete Finish energy system, this analysis is performed. 
Bioenergy, in particular, plays an important role in the future energy system and as stated in NETP 2013, the Nordic region becomes a net importer of bioenergy in CNS 2050 and imports $13 \%$ of its supply. Considering the import of bioenergy to Finland in CNS, an energy content of almost $40 \mathrm{PJ}$ is imported by 2050. Based on this assumption, import of bioenergy resources are allowed in the alternative transport scenarios, however they are compared with the national techno-economical available resources in Finland, which are estimated by VTT Finland and used in the NETP 2013 project.

Figure 2 presents the total use of resources in the Base year (use in 2012) and in CNS, EVS and BIOS by 2050 compared to the techno-economic potentials.

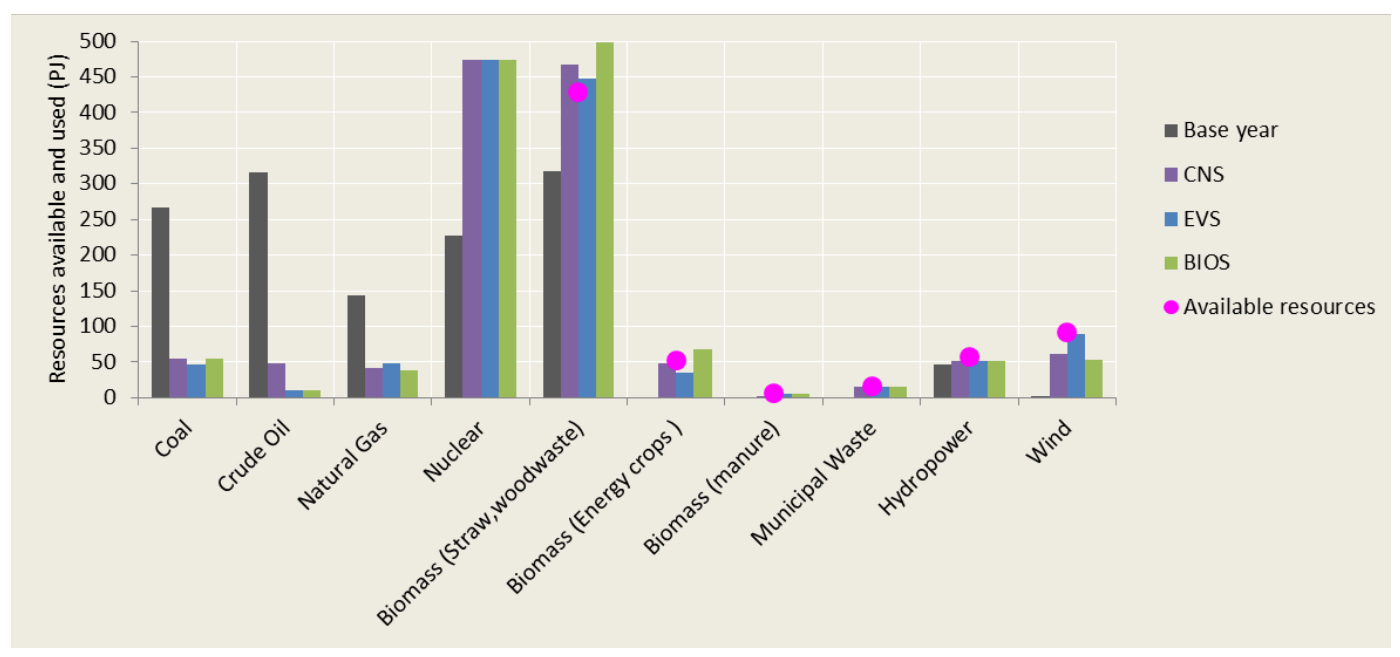

Figure 2: $\quad$ Resource use compared to available resources for the scenarios

Figure 2 shows the shift from the current fossil-fuel based energy system to an energy system which mainly relies on carbon neutral energy sources. The contribution of each fuel-type varies between the scenarios, however the use of bioenergy, wind and nuclear in the 2050scenarios accounts for a significant amount compared to the Base-year (2012).

In CNS, the Nordic region is assumed to become net importer of bioenergy i.e. straw \& wood waste, by 2050. Furthermore, the use of fossil fuels i.e. coal, oil and natural gas are still used to a small extent by 2050 .

In EVS, the use of wind energy is just below the techno-economical potential. Furthermore, bioenergy resources are used in all sectors, and Finland imports a small amount of straw \& 
wood waste. Moreover, the Finnish energy system is still dependent on fossil-resources by 2050.

BIOS is characterised by a large share of biofuel driven transportation which consequently causes a higher consumption of different types of bioenergy. Both straw \& wood and energy crops exceeds the techno-economical national available resources. According to the calculations, almost $70 \mathrm{PJ}$ of straw \& wood waste and $17 \mathrm{PJ}$ of energy crops need therefore to be imported by 2050 .

\section{Description of the Transport Systems}

Currently, the Finnish transport sector relies on fossil fuels as illustrated in Figure 3 which shows the allocation of fuel consumption in 2012 by vehicle types.

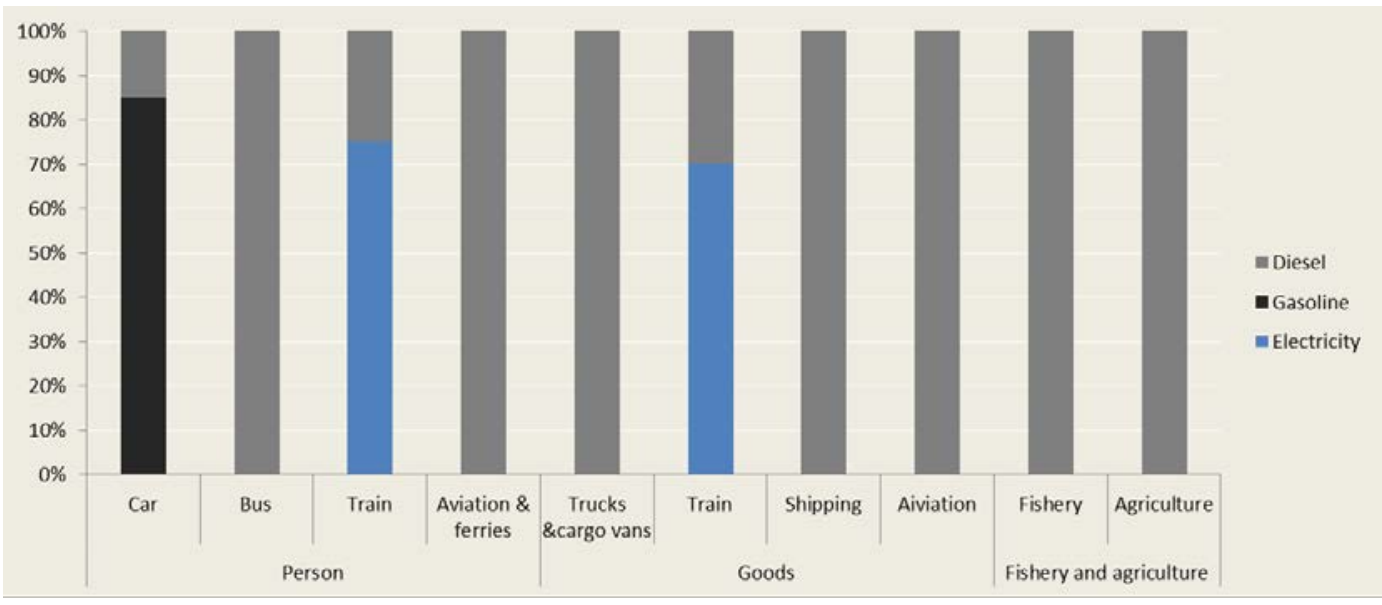

Figure 3: $\quad$ Fuel use in Finland (2012). Measured as fuel consumption in percent person transportation work (pkm) or percent freight transportation work (tkm)

Evidently, the fuel consumption is homogeneous and is dominated by oil-based fuels i.e. diesel and gasoline, which accounts $96 \%$ of the total final consumption in the transport sector. Diesel is the dominating fuel used for aviation, shipping, busses and cargo vans transportation, whereas gasoline is commonly used by cars where it accounts for $85 \%$ of the total fuel consumption in this sector. Electricity is used for train transportation, however the use of electricity in the others sectors are limited to a minor share of the total energy use. 
In order to meet the ambitious 2050-goal, radical restructuring of the fuel use and vehicle stock is required in the transport sector. Hence, the 2050-scenarios allocation of the fuel consumption is very diverse compared to the current situation. A careful description of the transport system for CNS, EVS and BIOS will be addressed in the following.

The projection for passenger and freight work by 2050 is based on CNS projections for all three transport scenarios. These projections are computed based on historical trends for transport work and economic growth along with assumptions for future transportation demand, transport work, efficiency improvements and technology switch.

The three transport scenarios vary in the composition of the fuel mix. The composition of the fuel mix in CNS is adopted from NETP 2013 and is shown in Figure 4.

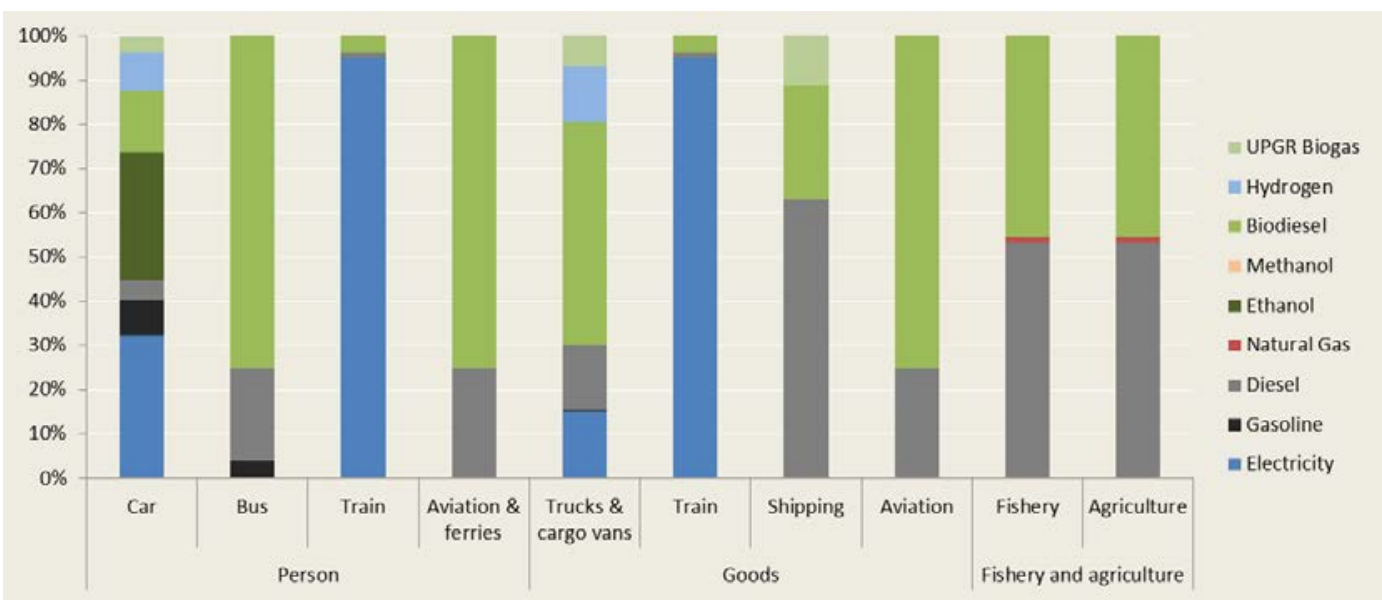

Figure 4: $\quad$ Fuel use in transport sector in CNS by 2050. Measured as fuel consumption in percent person transportation work (pkm) or percent freight transportation work (tkm)

In CNS, $32 \%$ of the fuel use for cars is electricity whereas only $4 \%$ and $8 \%$ of the fuel use is diesel and gasoline respectively. The rest of the fuel mix for cars will be diverse with $29 \%$ share of bio-ethanol, 14\% bio-diesel, 4\% UPGR bio-gas and 9\% hydrogen $\left(\mathrm{H}_{2}\right)$. Additionally, the composition in the other transport categories has much higher shares of electricity and biofuels. Within the biofuels, biodiesel is used as the main substitute for traditional diesel. 
In EVS a high share of EV is integrated. Figure 5 shows the fuel mix in the transport sector chosen for EVS.

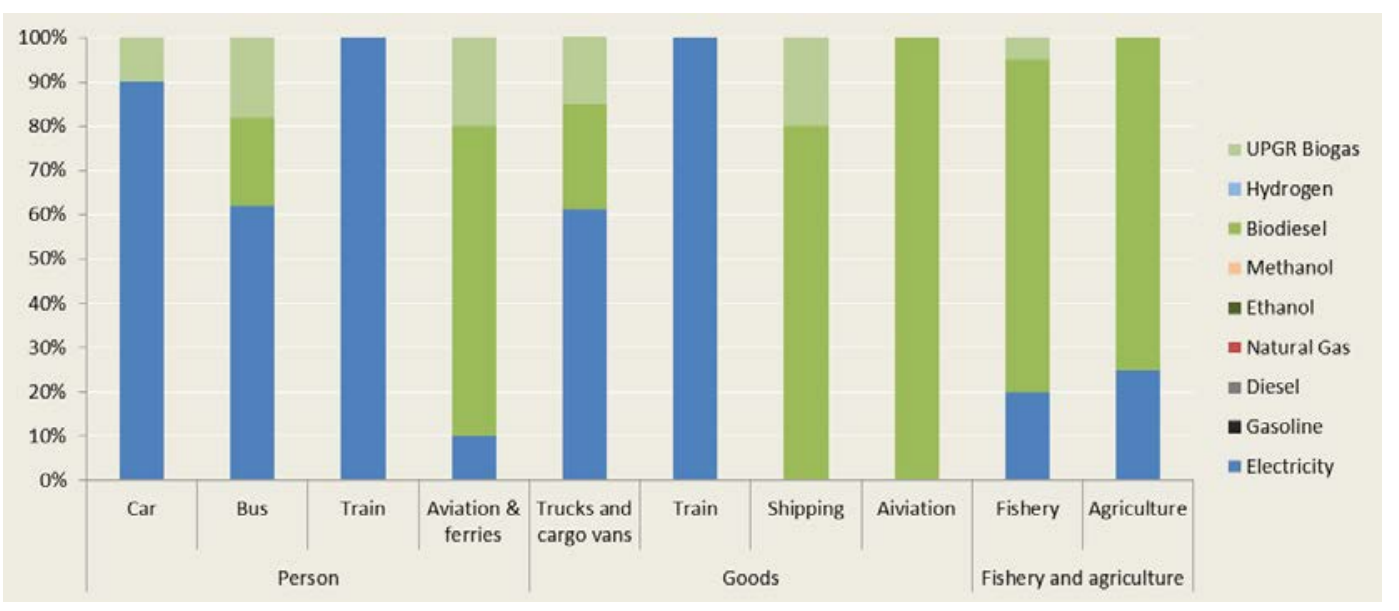

Figure 5: Fuel use in EVS by 2050. Measured as fuel consumption in percent person transportation work (pkm) or percent freight transportation work (tkm)

The electrification of the transport sector applied in EVS is illustrated in Figure 5, and shows that approximately $90 \%$ of the cars by 2050 are EV and all trains uses electricity. The bus, cargo vans and short to medium distance trucks sectors become more electrified in the future, however the remaining fuel used in these sectors are bio-fuels, mainly biodiesel and upgraded biogas. In the shipping and aviation sector, biodiesel is the preferred fuel.

In BIOS, high utilisation of biofuels is the way to a fossil free transport sector. In the development of the scenario, the same shares of EV in the fuel mix as in CNS are adopted. Therefore, biofuels cover the remaining share of transport demand, where biodiesel and upgraded (UPGR) biogas is the preferred fuel choices. Biodiesel chosen to be the key fuel in the future and UPGR biogas is used to the limit of the available resources.

The composition of the fuel use in BIOS is illustrated in Figure 6. 


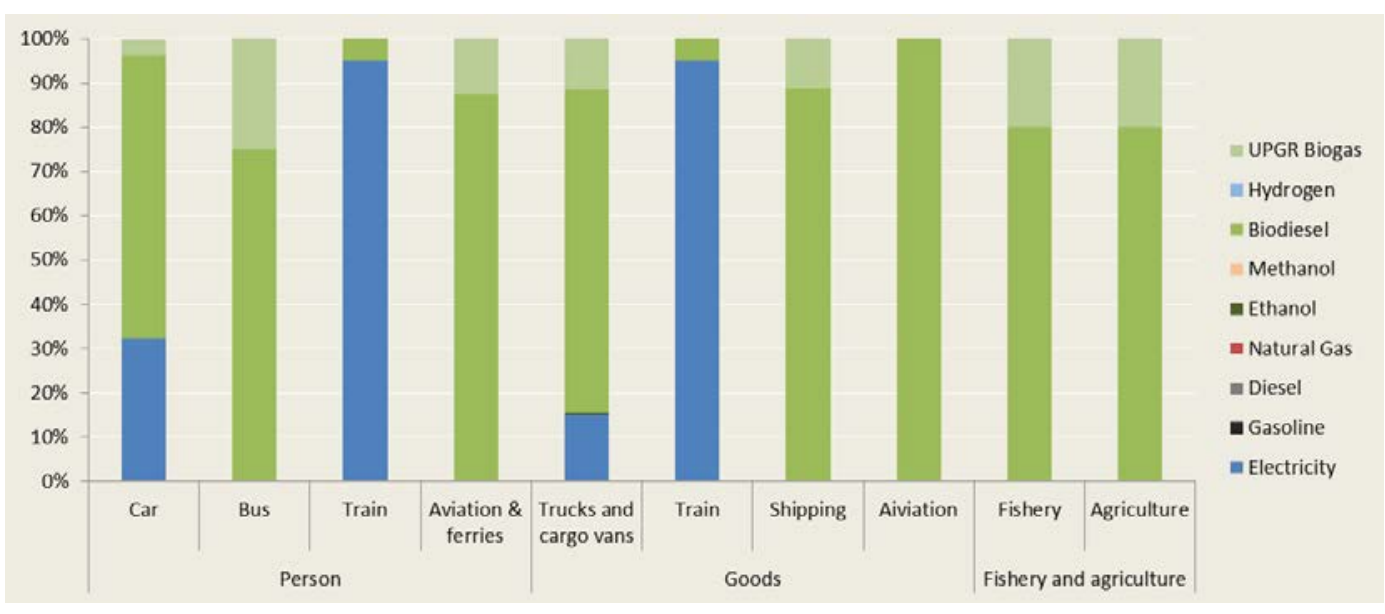

Figure 6: Fuel use in BIOS by 2050. Measured as fuel consumption in percent person transportation work (pkm) or percent freight transportation work (tkm)

As illustrated in Figure 6, the fuel mix in BIOS is more homogeneous compared to CNS. Biodiesel is diesel-equivalent and thus it is heavily used in the future transport sector. Biodiesel used for car transportation is $64 \%$ and $75 \%$ for busses. Considering the transportation of goods $89 \%$ of shipping and $100 \%$ aviation uses biodiesel as fuel. When considering the share of UPGR biogas in the passenger transport sector, $4 \%$ of all cars and $25 \%$ of all busses uses UPGR biogas. Furthermore, 11\% of cargo vans and short to medium distance trucks and shipping transport use UPGR biogas by 2050.

\section{Description of the Energy Systems}

To meet the ambitious 2050 target the remaining energy system is required to undergo a green transition. The electricity and district heating systems are designed according to the description in Section 3. Figure 7 illustrates the technology mix in the electricity supply sector in the three future scenarios compared to the technology mix in the base year. In the three future scenarios the total domestic electricity production is assumed to equal the domestic demand on a yearly basis, e.g. the yearly import equals export. However, hourly utilisation of transmission lines is allowed in STREAM and is used to flatten out excess or deficit electricity generation. 


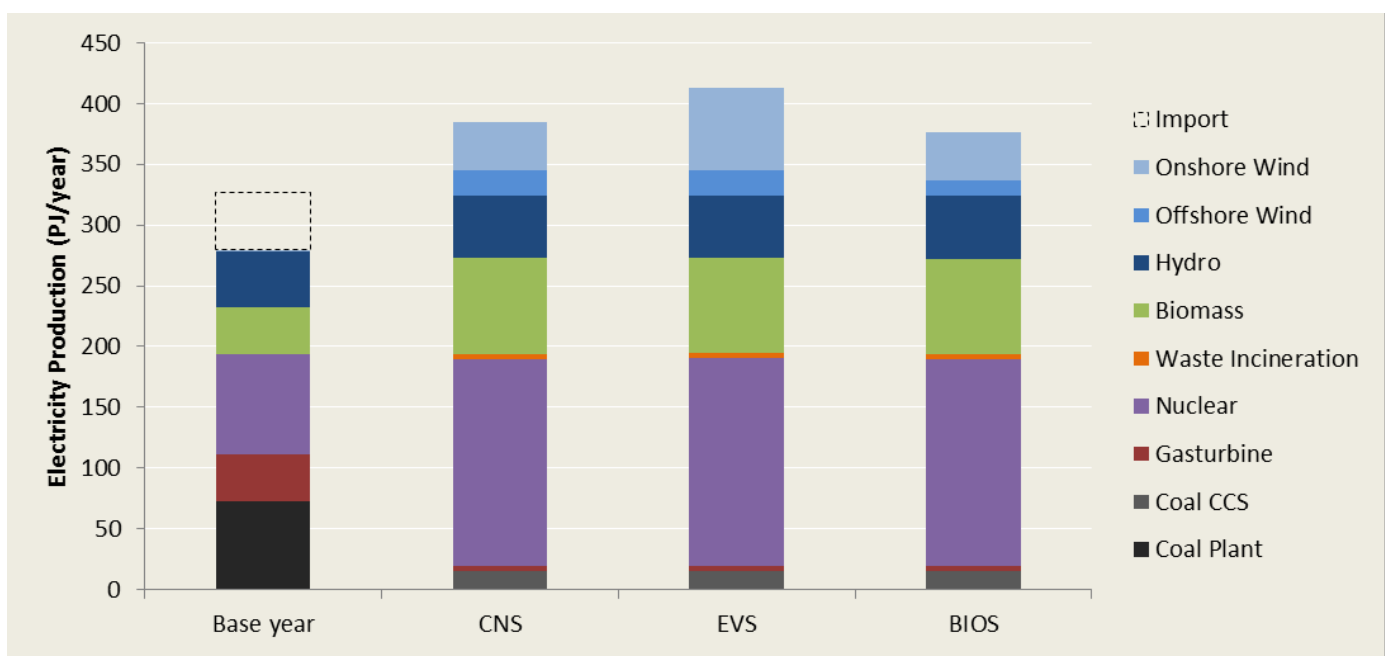

Figure 7: Technology mix in the electricity in three 2050 scenarios compared to the base year.

The Finnish electricity generation is, in the base year (2012), dominated by coal and nuclearpower plants, which accounts for $22 \%$ and $25 \%$ of the total electricity generation, respectively. Hydro, gas turbines and biomass based power generation have shares of $14 \%$, $12 \%$ and $12 \%$ respectively, and therefore are important technologies at present. It is notable that $14 \%$ of the total electricity consumed in Finland is imported from the neighbouring countries.

According to CNS, a considerable reduction in the use of coal and gas will be replaced by carbon neutral generation technologies. In Finland, nuclear power will be the major supplier of electricity by 2050, and Finland is expected to more than double the power generation from nuclear i.e. from 2.7 GW to $6.4 \mathrm{GW}$ by 2050 (IEA 2013a). Renewables will contribute to the electricity generation, where particularly the production from wind and biomass is expected to increase the contribution in the future. By 2050, electricity generated from wind turbines is 17 TWh and electricity produced using biomass as fuel is $21.8 \mathrm{TWh}$. Electricity production from hydro power plants is almost at the same level as today, while waste incineration utilises the full techno-economical potential to produce both heat and electricity, and therefore contributes with 1.1 TWh to the electricity production.

Figure 7 show how the metrics for the electricity production in CNS is adopted in the two alternative transport scenarios, with onshore and offshore wind generation as variable parameters to match the annual demand. A higher electricity production is found in EVS as a 
consequence of the higher penetration of $\mathrm{EV}$ and heat pumps for the purpose of individual heating. The electrification of the transport sector and consequently higher demand for electricity leads to $7 \%$ higher electricity production from the electricity generation technologies in EVS compared to CNS. In order to meet the higher demand, this implies an increment in the electricity generated by onshore wind power $7.8 \mathrm{TWh}$, which means a total wind production at $24.8 \mathrm{TWh}$ in EVS by 2050. In BIOS, the lower annual electricity demand compared to CNS is met by reduced offshore wind power production, as illustrated in Figure 7.

The CHP-biomass plants are running in backpressure mode and thus produce both electricity and heat. In STREAM, electricity production is modelled according to the electricity demand. Only as a consequence of this modelling structure, the heat production, which is associated with the electricity production appear due to the backpressure mode.

In 2050 the synergies between the transport, power and heating sectors are more coupled. Figure 8 compares the heating systems for the three future scenarios (CNS, EVS and BIOS) with the current situation.

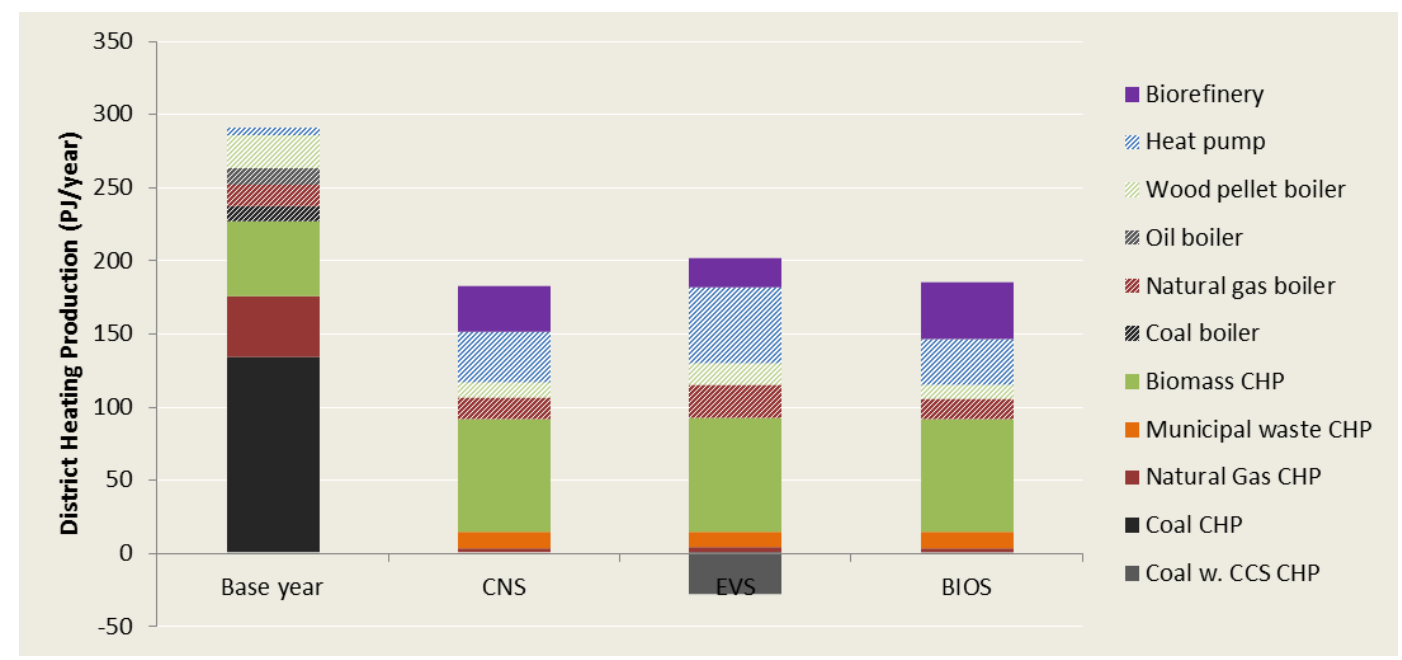

Figure 8: Technology mix in district heating in three 2050 scenarios compared to the base year.

The results regarding the district heating sector obtained by the model simulation is represented in Figure 8. The technologies used to produce heat for district heating by 2050 includes CHP plants, district heating boilers and excess heat from biorefinery processes. 
From investigation of the results, evidently the heat production from the CHP plants and the refinery processes are not enough to meet the heat demand without boiler usage for all the scenarios.

EVS has the largest heat production from district heating boilers at 89.6 PJ while the values are 59.8 and 54.4 in CNS and BIOS, respectively. These production levels are allocated with identical input of the percentage of the district heating boilers, namely $25 \%$ natural gas boiler, $58 \%$ heat pumps and 17\% wood pellets boilers in the STREAM model.

Heat pumps are introduced and allow utilising excess wind energy for producing heat. The highest utilisation of heat pumps appears in EVS where 52 PJ heat is produced. This result shows that EV together with heat pumps allows a larger integration of VRE e.g. wind energy.

Excess heat from biorefinery production can replace traditional heat supply. In BIOS, the highest contribution of heat from biorefinery is found. The heat production from biorefinery is $39 \mathrm{PJ}$ in BIOS while $31 \mathrm{PJ}$ and $20 \mathrm{PJ}$ are produced in CNS and EVS, respectively.

\section{Flexibility and system costs}

In general, the electricity generation portfolio by 2050 primarily relies on dispatchable power plants i.e. nuclear, coal, biomass and hydro. The shares of non-dispatchable VRE generation i.e. wind power, vary between the 2050-scenarios and are $21.6 \%$ in EVS, $15.9 \%$ in CNS and $14 \%$ in BIOS. The increased implementation of VRE, which has a stochastic electricity production profile, requires a flexible energy system in order to meet the associated challenges. In Finland, the integration of wind power is facilitated by the flexibility that hydro power reservoirs can provide and the transmission capacity to the surrounding countries $^{1}$. However, the implementation of a large share of EV can provide additionally demand-side flexibility into the energy system.

\footnotetext{
${ }^{1}$ This analysis investigate Finland as operating in an island mode
} 


\section{Flexibility added to the system}

Figure 9 illustrates the electricity generation and consumption in EVS. The production is represented by the different electricity generation technologies in order to meet the required electricity demand. In the figure the demand is represented by both the domestic electricity demand (green) and the electricity consumption including flexibility (cyan).

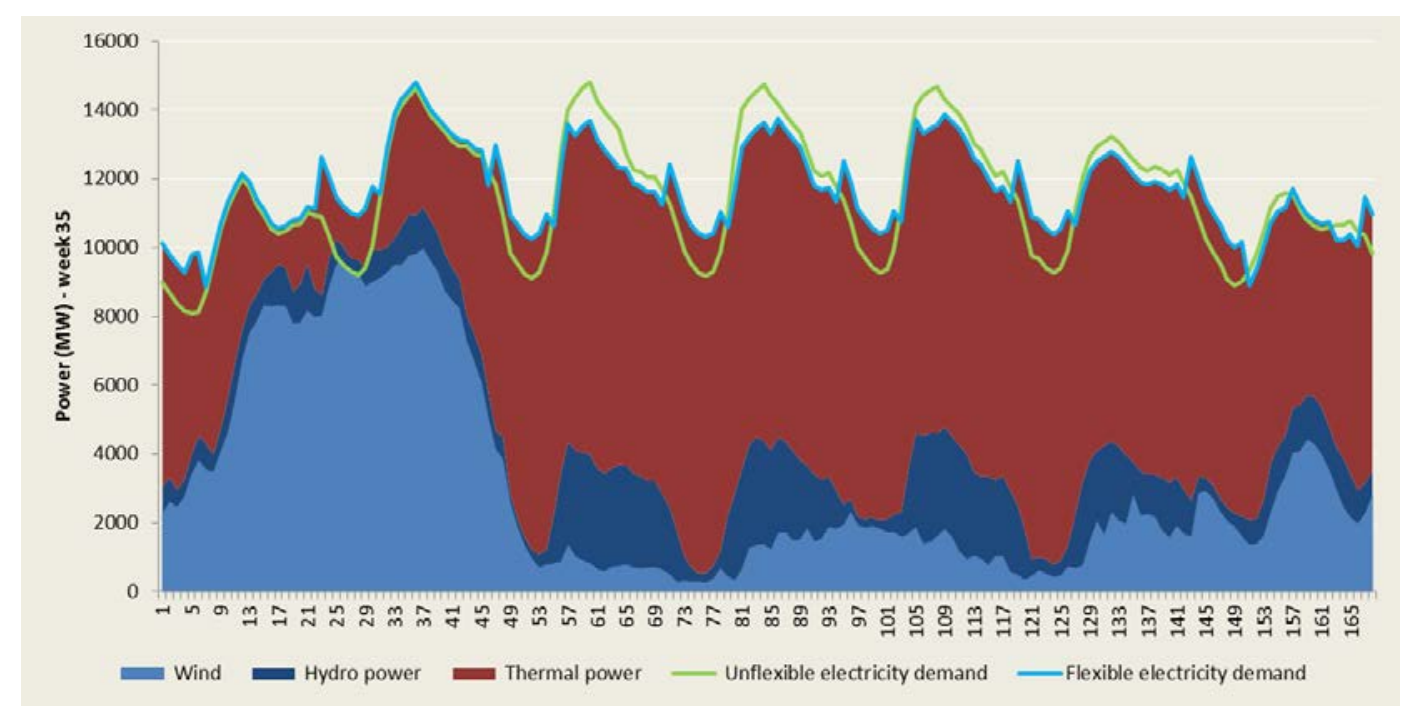

Figure 9: Electricity generation and consumption in EVS with and without flexible demand in week 35 in year 2050. Model simulation.

In the figure, the different electricity generation technologies are represented by different colour codes. Evidently, the wind power production in Finland can cover a high share of the consumption in time periods with high availability of wind, however as illustrated in the figure the wind power production can be low in other time periods.

The transport sector can provide demand-side flexibility into the electricity system by charging the EV utilising the concept of G2V. During night hours, where the traditional electricity consumption is low, EV can be charged and thereby move a part of the electricity consumption between the hours. In the STREAM simulation of the electricity consumption including flexibility, $40 \%$ of all EV are assumed to be charged in a flexible way in order to provide flexibility best for the system. Furthermore, additionally $40 \%$ of the EV is assumed to be charged during night time (23h-6h). Hence, during night maximum $80 \%$ of EV can deliver a flexible electricity demand when charging the EV. 
The purpose of integrating flexible charging EV into the electricity system is to increase or reduce the consumption in time periods with high or low generation from VRE technologies. In Figure 9, the model increase the electricity demand in time periods with high shares of wind production and lower the electricity demand in hours with less VRE production. With increased demand-side flexibility, the system-integration of a high penetration of VRE can be facilitated in a cost efficient way that keep the cost for backup capacity down.

To keep the cost for backup capacity down, the model uses the flexible demand to minimise the thermal capacity i.e. the difference between the demand and the VRE production (red area) for each hour. During day time hours, which is characterised by high traditional electricity consumption, the demand flexibility from the EV reduces the demand in order to have less production from thermal power plant and thus less need for thermal generation capacity.

Since the charging of EV is more flexible during night hours, unusual demand peaks appear which indicates the demand shift from daytime to the night hours.

In BIOS, the contribution of demand side flexibility added to the system is limited, due to the lower amount of EVs. The result of the hourly electricity generation and consumption from the model simulation of BIOS is illustrated in Figure 10.

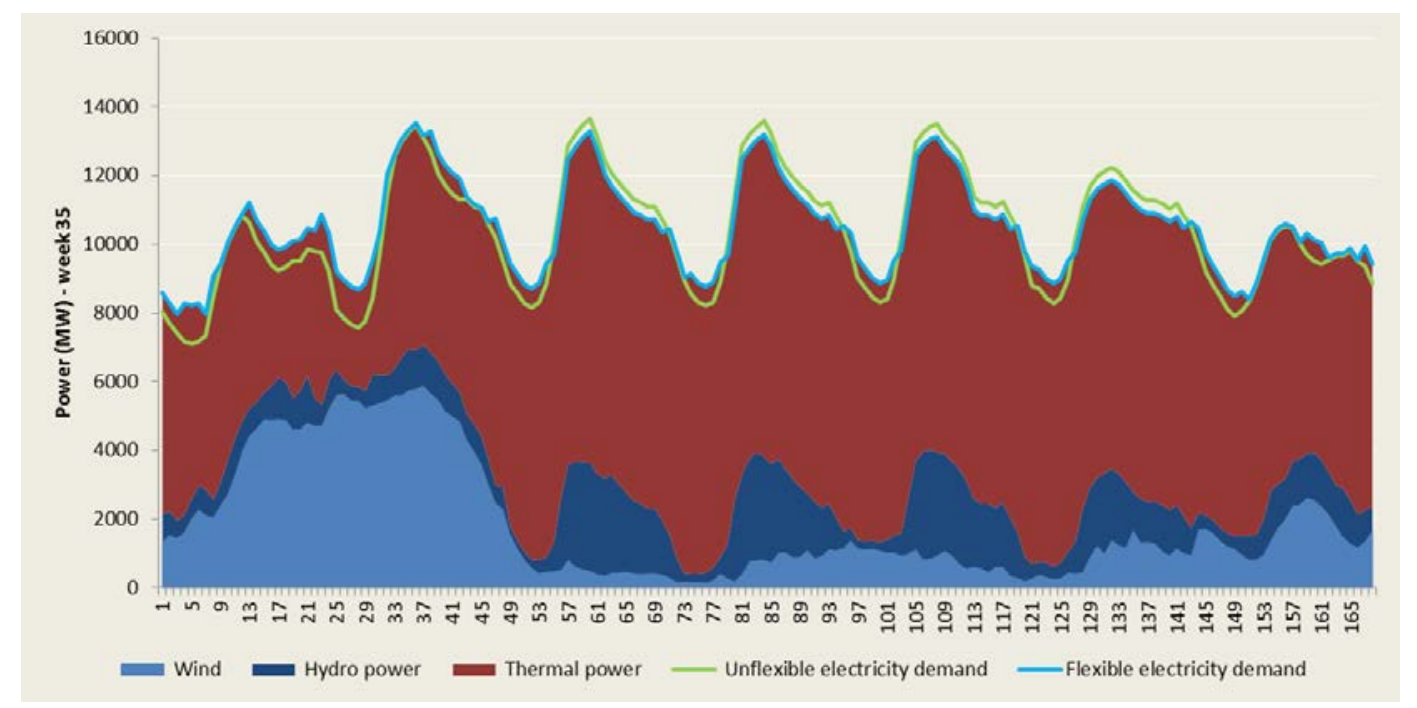

Figure 10: Electricity generation and consumption in BIOS with and without flexible demand in week 35 in year 2050. 


\section{Systems costs}

The STREAM model computes the socio-economic cost of the entire energy system. Figure 11 presents the total annual system costs obtained by the model simulations. To identify the drivers for discrepancies in the system costs between the scenarios, the total annual cost for the entire energy system is disaggregated into: Cost of Energy Savings, Capital Cost, O\&M Cost, Fuel Cost and $\mathrm{CO}_{2}$ Cost.

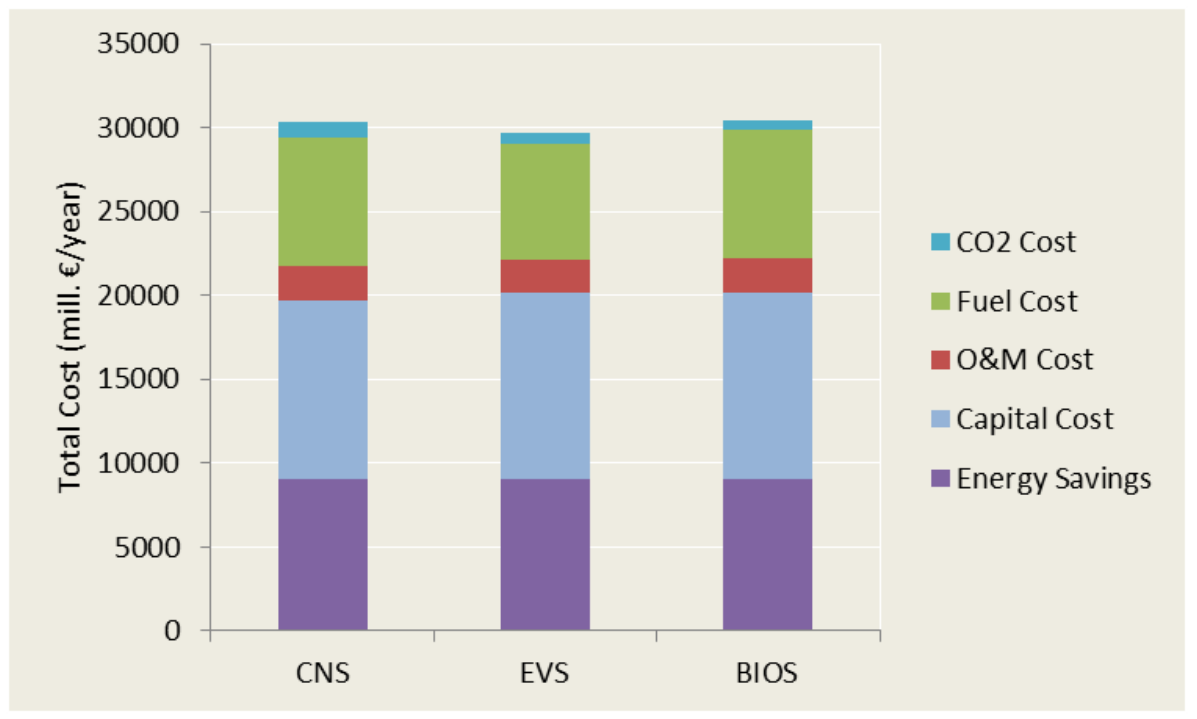

Figure 11: Total annual system cost in the three scenarios by 2050.

The STREAM runs find the total annual system cost in CNS to 30358 mill. $€$. By using CNS as a reference, the model computes the total annual system cost in EVS to be 29659 mill. $€$, which corresponds to a reduction at $2.3 \%$. BIOS is determined to be the most expensive with a value of 30480 mill. $€$ corresponding to an increment at $0.4 \%$ compared to CNS.

The quantification of the total annual system costs allows for a comparative analysis of the differences in the total system costs between the alternative transport scenarios and CNS, which is illustrated in Figure 12. 


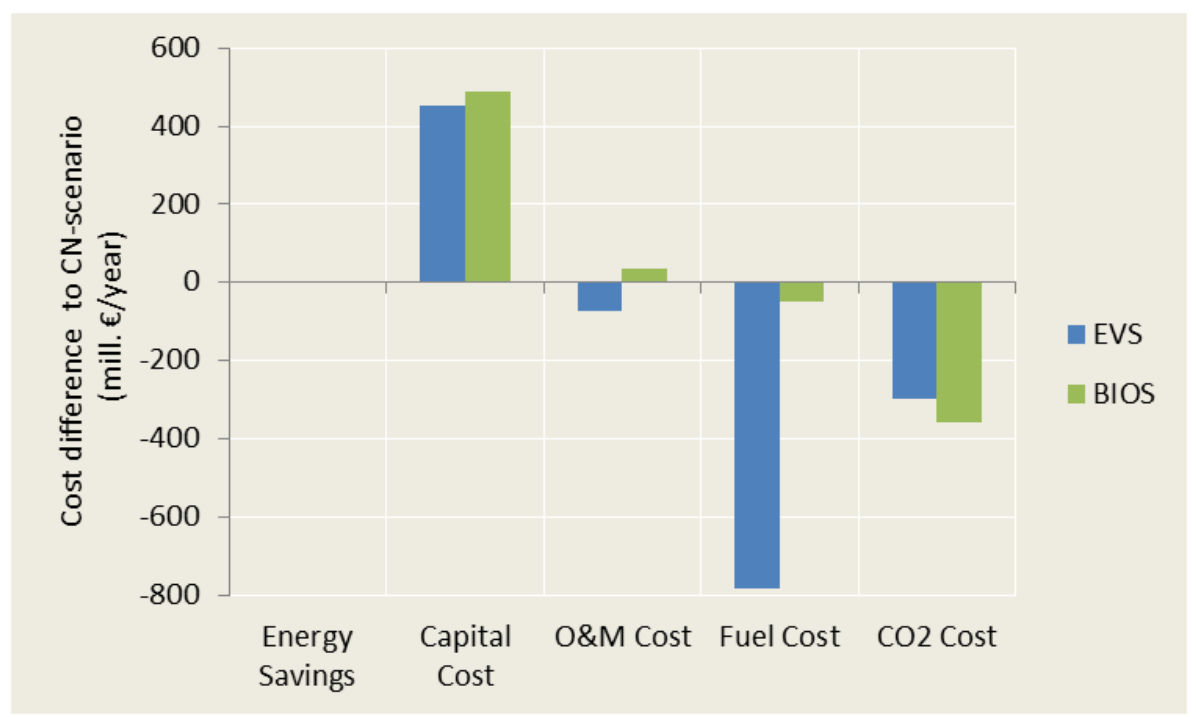

Figure 12: Cost difference in total annual system cost by 2050 between the two alternative transport scenarios and CNS.

In the following section, the main drivers for the differences in the system cost will be identified and further evaluated. EVS is the cheapest scenario, due to low fuel and $\mathrm{CO}_{2}$ costs, however the capital costs are higher than in CNS. The total increment in capital cost is 452 mill $€$ in EVS. By disaggregating this value into sectors, the main driver to the increased total capital cost in EVS is identified to be in the electricity and CHP generation technologies which cause an increased capital cost at 495 mill. $€$. The total annual capital cost in the transport sector is increased by 5\%. The capital cost of driving EV is assumed to be more than three times more costly than traditional gasoline vehicles when charging stations and rental of batteries are included in the capital costs. The electric cars are furthermore assumed to be cheaper than the biofuel substitute and thus lower the increase in capital costs from the transport sector.

The increased electrification of the transport sector consequently leads to higher electricity demand. In order to meet the increased electricity demand, more capacity in the electricity system is needed. Since onshore wind production is increased according to the increased electricity demand, the capital cost in the electricity sector is increased. The large deployment of wind energy in EVS leads to higher capital cost for wind energy. Furthermore it demands increased backup capacity such as natural gas turbines. This subsequently implies increased capacity of natural gas turbines and thus increases the capital costs in EVS compared to CNS. 
The results in Figure 12 show a significant reduction in the costs related to the fuel in EVS. The main driver of reduced fuel cost in EVS compared to CNS is the costs related to the fuel used in the refinery processes. The model finds $81 \%$ higher fuel costs in the refinery processes in CNS compared to EVS. In total this implies a large saving in fuel costs, even though the model finds approximately 50\% higher fuel cost for district heating production technologies in EVS compared to CNS, due to higher demand for district heating boilers.

The model results indicate that BIOS is the scenario that has the highest total annual system cost. The increment in the capital cost compared to CNS is 489 mill. €, corresponding to an increase at $10 \%$, appear mainly due to high investment costs in biofuel transportation. The capital cost for bioenergy transportation is increased by approximately $60 \%$ compared to CNS. The $\mathrm{CO}_{2}$ cost is reduced in BIOS compared to CNS. In CNS, fossil fuels are still used in the transport sector and thereby are related to a $\mathrm{CO}_{2}$ cost.

\section{Clarify sensitivities of main assumptions}

The robustness of the results obtained in the quantitative assessment will be tested by separately varying four parameters i.e. prices of bioenergy, cost (investment and maintenance) of cars both for $\mathrm{EV}$ and biodiesel, and the $\mathrm{CO}_{2}$ price, and evaluate the effects on the total annual system cost.

STREAM is a simulation tool and therefore changes in prices will not influence the composition of technologies in the sectors. By varying the input prices, the total annual system cost will change with a constant slope. The percentage change caused by changing the parameters 1\% is illustrated in Figure 13. 


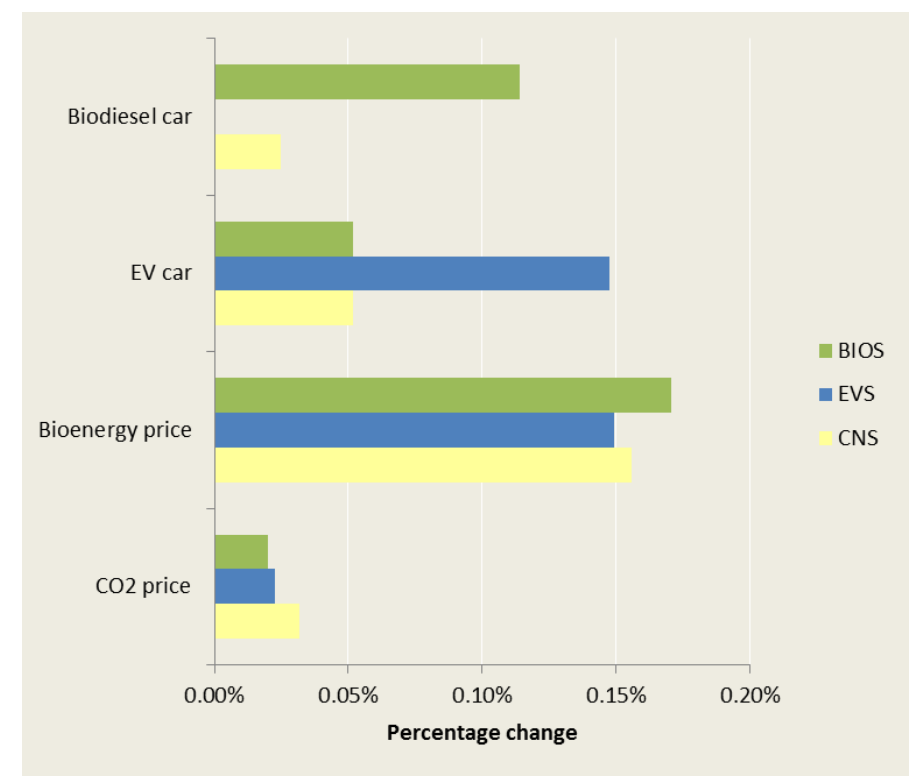

Figure 13: Sensitivity analysis for the three 2050 scenario. The figure show the percentage change caused by changing the parameters $1 \%$.

The sensitivity analysis shows that the total annual system cost is most sensitive to changes in the prices of bioenergy resources for all three scenarios. The sensitivity to the costs (investment and maintenance) of the car types varies significant between the scenarios. BIOS is sensitive to changes in costs of biodiesel cars, while EVS is sensitive to changes in costs of $\mathrm{EV}$. As CNS has the highest emission of $\mathrm{CO}_{2}$, CNS is most sensitive to changes in $\mathrm{CO}_{2}$ prices, however the percentage change in the total annual system costs is rather small for all scenarios.

Since this study compare two alternative transport scenarios with CNS, it is valuable to perform a threshold analysis. In Figure 14, the parameters are varied $\pm 50 \%$ compared to their original value, however as the implemented $\mathrm{CO}_{2}$ price is corresponding the ambitious 2DS and is rather high, the sensitivities related to the $\mathrm{CO}_{2}$ price is tested from the original value to $-50 \%$. 


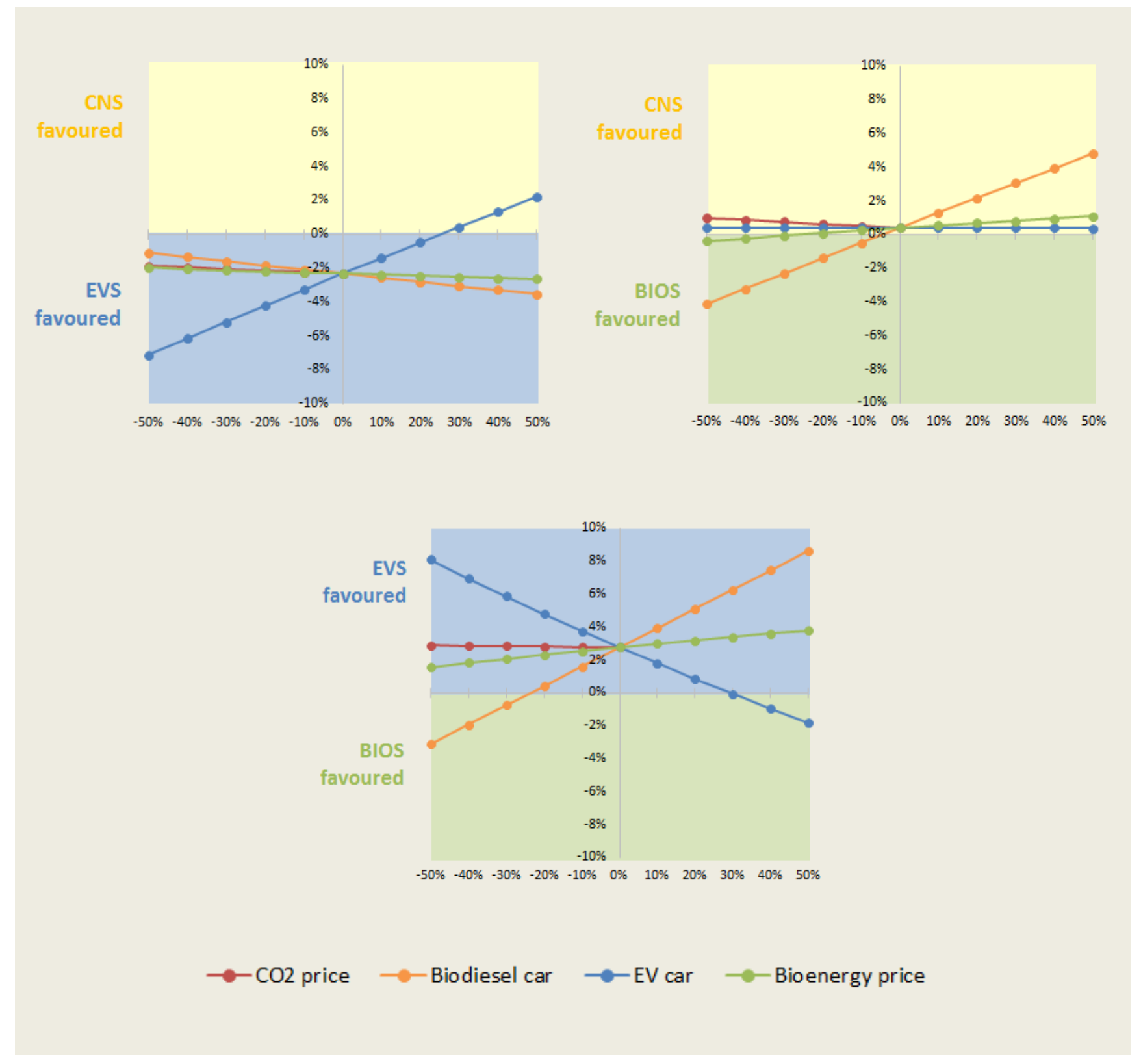

Figure 14: Threshold analysis which points out the scenario which achieves the lowest total annual cost for the entire energy system by 2050.

Comparing EVS with CNS, only 30\% higher costs related to the EV causes a shift so CNS achieves the lowest total annual system cost. Even though the bioenergy price was the most sensitive parameter, it affects both scenarios and thus only to a small extent influence the price difference between the scenarios.

BIOS has a total annual system cost which is $0.4 \%$ more expensive compared to CNS. However, in case the biodiesel cars can achieve10\% lower costs, BIOS perform better than 
CNS in terms of total annual system cost. Furthermore, if the cost of biodiesel cars reduced by $50 \%$ BIOS achieves a result which is $4 \%$ better than CNS. High utilisation of bioenergy appear in both scenarios, however BIOS may perform better in case the bioenergy prices reduced by $30 \%$ and more.

Comparing the two alternative transport scenarios, it can be observed that the costs of cars is the parameter which can make BIOS better performing compared to EVS. In case either the cost of biodiesel cars reduces by $30 \%$ or the cost of $\mathrm{EV}$ is more than $30 \%$, BIOS can achieve lower total annual system costs compared to EVS.

\section{Conclusion}

This study investigated the long-term role of EV or biofuels in facilitating the decarbonisation of the Finnish transport sector by 2050. By utilising a holistic energy system perspective, the transport sector was elucidated while taken into account future synergies between the power and heating sectors. The energy system model, STREAM, computed the socio-economic cost of the system and simulated the system integration of the transport sector with the electricity and heating sectors.

This paper compared a known scenario (CNS) adopted from Nordic Energy Technology Perspective 2013 with two alternative transport scenarios - either with a high percentage of EV (EVS) or with a high percentage of biofuel use (BIOS) in the transport sector.

The result shows that a Finnish transport sector with a high share of EV by 2050 leads to the cheapest cost solution under the given assumptions and yields a reduction in the total annual system cost by $2.3 \%$ compared to CNS. While the transport configuration in BIOS achieves the highest total annual system cost which is $0.4 \%$ higher than CNS.

Even though large bioenergy resources appear in Finland, the use of bioenergy resources scenarios exceed their available domestic resources. The market price of bioenergy resources depend on the utilisation worldwide. Through a sensitivity analysis, the robustness of the scenario results was tested by changing bioenergy prices. Each of the scenarios was highly sensitive to changes in bioenergy prices, however introducing identical bioenergy prices in all scenarios, the results did not lead to different best performing solutions. 
Biodiesel cars and EV are key technologies in the two alternative transport scenarios. It was shown that the scenario results were sensitive to changes in the costs (investment and maintenance) of these vehicle types and therefore show the importance of the future development of these two vehicle technologies. By reducing the costs of biodiesel cars by around 30\% while keeping other input parameter constant, BIOS will be the scenario which achieves the lowest total annual system cost by 2050. Furthermore, by increasing the costs of EV by approximately 30\%, EVS will no longer achieve the lowest total annual system cost. 


\section{References}

Connolly, D., Lund, H., Mathiesen, BV., Leahy, M., (2010) A review of computer tools for analysing the integration of renewable energy into various energy systems. Applied Energy, 87, pp 1059 - 1082.

EA (2013), EA Energy Analyses for Danish Energy Agency. Analysis of biomass prices Future Danish Prices for Straw, Wood chips and wood pellets. [Bang, C., Vitina, A., Sterling Gregg, J. and Lindboe, HH.]

EC (2011), European Commission, A Roadmap for moving to a competitive low carbon economy in 2050. Communication from the Commission to the European Parliament, the Council, the European Economic and Social Committee and the Committee of the Regions.

EC (2009), European Commission , Directive 2009/28/EC of the European Parliament and of the Council of 23 April 2009 on the promotion of the use of energy from renewable sources and amending and subsequently repealing Directives 2001/77/EC and 2003/30/. Official Journal of the European Union, L 140/16-L 140/61. 2009.

DEA (2013), Danish Energy Agency \& COWI, Alternative drivmidler i transportsektoren 2.1. May 2013.

DEA (2012), Danish Energy Agency \& Energinet.dk, Technology Data for Energy Plants Individual Heating and Energy Transport. May 2012.

FMEE (2014), Finish Ministry of Employment and the Economy, Energy and Climate Roadmap 2050, Report of the Parliamentary Committee on Energy and Climate Issues on 16 October 2014.

Börjesson, M., Grahn, M. \& Ahlgren, E. O. (2013) Transport Biofuel Futures in EnergyEconomic Modeling - A Review. Report No 2013:10, f3 The Swedish Knowledge Centre for Renewable Transportation Fuels, Sweden. 
IEA (2013a), International Energy Agency \& Nordic Energy Research. Nordic Energy Technology Perspective - Pathways to a Carbon Neutral Energy Future.

IEA (2013b), International Energy Agency, Energy Policies of IEA Countries, Finland, 2013 review.

IEA (2012), International Energy Agency, Energy Technology Perspectives 2012 - Pathways to a Clean Energy System.

Juul, N. \& Meibom, P. (2011) Optimal configuration of an integrated power and transport system. Energy, 36, pp 3523 - 3530.

Kempton, W. \& Tomic, J. (2005) Vehicle-to-grid power fundamentals: calculating capacity and net revenue. Journal of Power Sources, 144, pp, 268-279.

Kiviluoma, J. \& Meibom, P. (2010) Influence of wind power, plug-in electric vehicles, and heat storages on power system investments. Energy, 35, pp 1244-1255.

Münster, M., Larsen, H., Iversen, J. (2014) - DTU Risø and EA Energy Analysis, STREAM (Sustainable Technology Research and Energy Analysis Model) Manual. August 2014.

Skytte, K., Meibom, P., Henriksen, TC. (2006) Electricity from biomass in European Union With or without biomass import. Biomass \& Bioenergy, 30, pp 385-392.

Skytte, K. \& Skjerk Christensen, P. (1999) An energy system simulator for long term planning. Revista Mexicana de Fisica, 45(5), pp 532-538. 